\title{
PRÁCTICAS DE CAZA: ANÁLISIS DE MANDÍBULAS Y MAXILARES DE GUANACO DEL SITIO CERRO CASA DE PIEDRA 7 (SANTA CRUZ, ARGENTINA)
}

NATALIA L. FERNÁNDEZa \& MARIANA E. DE NIGRIS

\begin{abstract}
RESUMEN
El objetivo del presente trabajo es discutir las prácticas de caza implementadas por los grupos cazadores recolectores que ocuparon el sitio Cerro Casa de Piedra 7, Parque Nacional Perito Moreno (Santa Cruz, Argentina), en el Holoceno medio. Los trabajos zooarqueológicos previamente realizados en el sitio mostraron que el guanaco (Lama guanicoe) era la presa principal, sin embargo, dado que dichos estudios se han centrado en el procesamiento y consumo de los recursos aportados por esta especie, no se han evaluado hasta el momento las diversas formas empleadas para su adquisición. La construcción de perfiles de mortalidad, a través del análisis de mandíbulas y maxilares, es una vía útil para abordar la problemática de la selección de presas. Las muestras consideradas provienen de dos áreas de excavación diferentes datadas en ca. 5.000 años AP, momento caracterizado por una mayor redundancia y/o intensidad de las ocupaciones en el Parque Nacional Perito Moreno y áreas vecinas. Los resultados obtenidos muestran que hubo marcada selección de individuos en edad reproductiva - 2 a 8 años aproximadamente-, posiblemente procedentes de grupos familiares y capturados durante todo el ciclo anual.
\end{abstract}

PALABRAS CLAVE: guanaco, zooarqueología, perfiles de mortalidad, Patagonia, Holoceno medio.

\section{HUNTING PRACTICES: GUANACO'S MANDIBLES AND MAXILLAS ANALYSIS FROM CERRO CASA DE PIEDRA 7 SITE (SANTA CRUZ, ARGENTINA)}

\section{ABSTRACT}

The aim of this paper is to discuss hunting practices implemented by hunter-gathered groups that inhabited Cerro Casa de Piedra 7 site, Perito Moreno National Park (Santa Cruz, Argentina) during Middle Holocene. Previous zooarchaeological studies conducted in the site have shown that guanaco

a Agencia Nacional de Promoción Científica y Tecnológica-Instituto Nacional de Antropología y Pensamiento Latinoamericano. 3 de Febrero 1370 (C1426BJN), Ciudad Autónoma de Buenos Aires. natalialuciafernandez@yahoo.com

b Consejo Nacional de Investigaciones Científicas y Técnicas-Universidad de Buenos Aires/Instituto Nacional de Antropología y Pensamiento Latinoamericano. 3 de Febrero 1370 (C1426BJN), Ciudad Autónoma de Buenos Aires. mariandenigris@yahoo. com.ar 
(Lama guanicoe) was the main prey, however these studies have been centered in processing and consumption practices of this camelid. The way of capturing this prey has not been evaluated until now. Mortality patterns, based on mandibles and maxillas analysis, are useful to address prey selectivity issue. The selected samples come from two different areas of excavation dated ca. 5,000 BP, time characterized by a great occupational redundancy in Perito Moreno National Park and neighbor areas. Results show a prime dominated -2 to 8 years old approximately- mortality profile. Guanacos might have been hunted out of family groups during all year round.

KEY WORDS: guanaco, zooarchaeology, mortality patterns, Patagonia, Middle Holocene.

\section{INTRODUCCIÓN}

El guanaco (Lama guanicoe) ha sido, sin lugar a dudas, la principal presa de las poblaciones cazadoras recolectoras que habitaron el interior de Patagonia durante el Holoceno (Mena \& Jackson, 1991; Gradin \& Aguerre, 1994; Miotti, 1998; Mengoni Goñalons, 1999; Silveira, 1979; entre otros). Su rol destacado ha motivado una serie de estudios que se centran en dos aspectos principales, los tipos de transporte implementados (e.g. Mengoni Goñalons, 1999; Miotti et al. 1999; Rindel 2003; Savanti et al. 2004), y las prácticas utilizadas para procesar y consumir este camélido (Mengoni Goñalons, 1999; De Nigris \& Catá, 2005; De Nigris, 2008; Marchionni et al. 2010 , entre otros). Para abordar estos temas las investigaciones se basaron fundamentalmente en los patrones de representación anatómica y en las modificaciones que exhiben los especímenes óseos (e.g. Mengoni Goñalons, 1999; Bourlot, 2009; Bourlot et al. 2009; Méndez et al. 2011; De Nigris \& Tecce, 2013).

Los análisis efectuados en los conjuntos zooarqueológicos procedentes del Parque Nacional Perito Moreno (PNPM), emplazado el noroeste de Santa Cruz, no escapan a estas tendencias generales (e.g. Cassiodoro et al. 2000; De Nigris, 2000, 2004, 2007; Rindel, 2003, 2004, 2008, 2013). Si bien estas propiedades resultan relevantes para comprender la naturaleza de la relación entre los humanos y el guanaco en el pasado existen otros atributos que pueden aportar información importante para profundizar en este vínculo, tal es el caso de los perfiles de mortalidad. Esta herramienta metodológica es considerada útil para discutir las diferentes formas de adquisición desarrolladas por los grupos cazadores recolectores (e.g. Mengoni Goñalons, 1988; Stiner, 1990;
Lyman, 1994; Legge \& Rowley-Conwy, 1997; Kaufmann, 2004, 2009; Atici, 2009).

Es notable que a pesar de su gran potencial poco se ha hecho hasta el momento en esta región, es por ello que en el presente trabajo se propone el análisis de mandíbulas y maxilares recuperados en el sitio Cerro Casa de Piedra 7 (CCP7) con la intención de construir perfiles de mortalidad. Las muestras seleccionadas proceden de dos sectores distintos de excavación, fechadas en torno a los 5.000 años AP.

Este lapso temporal resulta particular dado que se observó un notorio incremento en el número de las dataciones disponibles en el PNPM. El aumento en la cantidad de fechados se ha visto reflejado tanto en la ocupación de nuevos espacios como en la reocupación, con mayor frecuencia, de sitios preexistentes (Aschero, 1996; De Nigris, 2004; Aschero et al. 2005). La evidencia es concordante con lo observado en áreas vecinas del centro-sur y este de la cuenca de los lagos Pueyrredón y Posadas, el lago Salitroso y el área del río Pinturas donde, del mismo modo, se registra un aumento en la intensidad de ocupación de los sitios (e.g. Gradin \& Aguerre, 1994; Aschero, 1996; Aschero et al. 2009). A nivel regional, también, se observa esta tendencia en una intensificación creciente en el uso del espacio que se manifiesta en la cantidad de sitios, en el número de hallazgos aislados y/o en una alta redundancia de ocupación. Las interpretaciones al respecto de este particular uso del espacio han sido variadas, entre las cuales se incluyen una reestructuración de los recursos de subsistencia (Mancini et al. 2002) y/o un incremento de la población (e.g. Yacobaccio \& Guráieb, 1994; Aschero et al. 2005; Miotti, 2006). Precisamente, las evidencias recuperadas en el sitio CCP7 irían en esta dirección. Una serie de fechados radiocarbónicos, obtenidos en el 


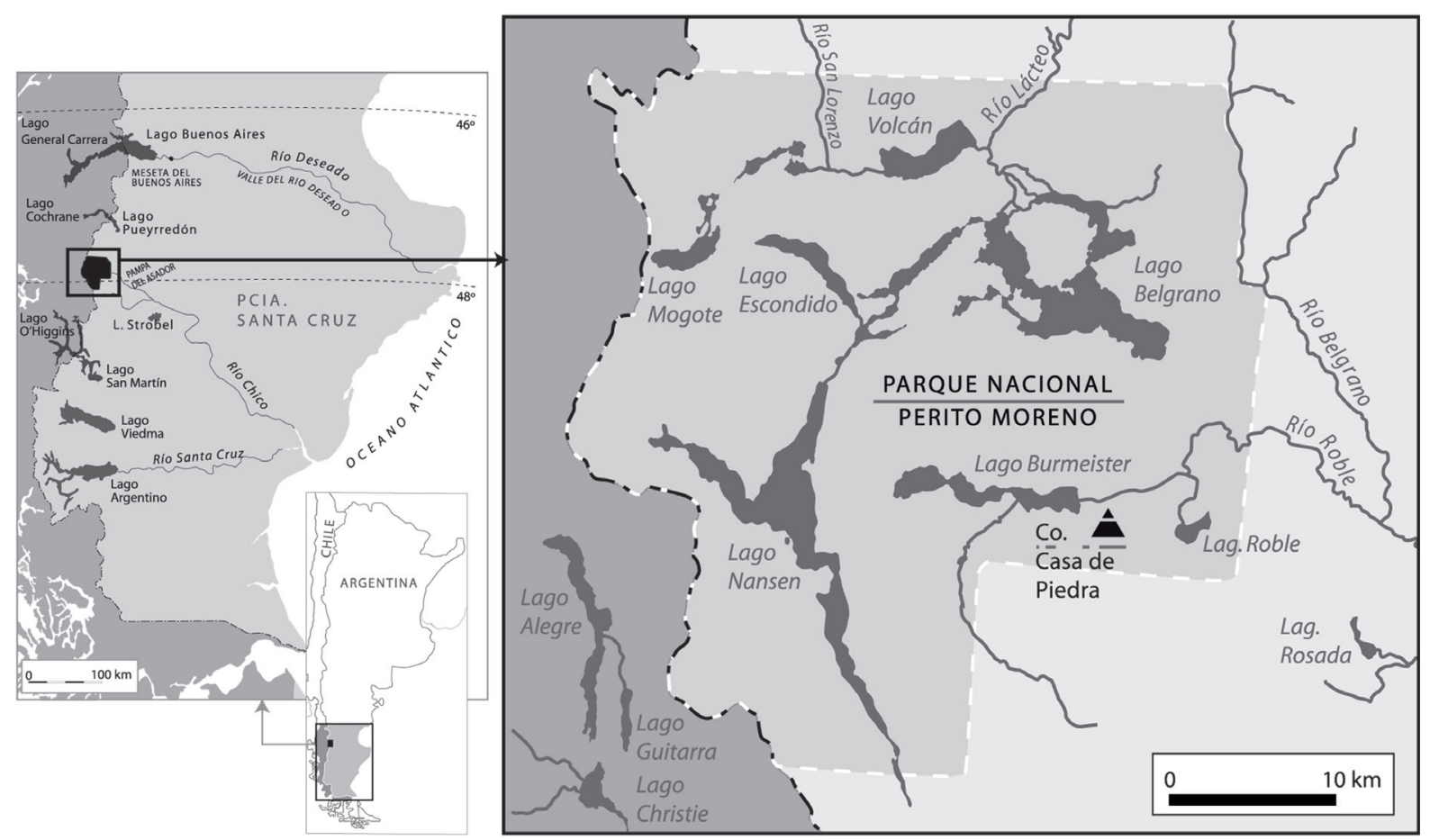

Fig. 1. Localización del Parque Nacional Perito Moreno y Cerro Casa de Piedra.

nuevo sector excavado, pertenecientes al Holoceno medio estarían señalando una mayor redundancia en el uso de este sitio. De esta forma, las muestras óseas recuperadas nos permitirán observar ciertas características de los aspectos organizativos relacionados con las actividades de subsistencia, en este caso particular, sobre ciertos aspectos de las prácticas de caza de los guanacos.

Dado que el guanaco es la presa de mayor porte disponible y la más abundante debido a la baja diversidad estructural del hábitat (Redford \& Einsenberg, 1992) nos planteamos como expectativa que en estos momentos de mayor redundancia en la ocupación los cazadores no hayan sido selectivos y que camélidos de todas las edades hayan sido capturados, ejerciendo de esta forma una mayor presión sobre los grupos familiares.

\section{DESCRIPCIÓN DEL SITIO Y LAS ÁREAS DE EXCAVACIÓN}

La localidad de Cerro Casa de Piedra se ubica en la cuenca del lago Burmeister y el río Roble, en la zona cordillerana del PNPM, en el noroeste de la provincia de Santa Cruz. Sobre el frente norte de un cerro homónimo se emplazan una serie de cuevas y aleros, entre las cuales destaca la Cueva 7 por ser la que presenta la secuencia estratigráfica más larga de la región y la única con evidencias datadas en el Holoceno temprano (Aschero et al. 1992, 1992-1993, 2005, 2007; Fig. 1).

Las investigaciones paleoambientales señalan que a partir de ca. 9.000 años AP una importante disminución en las proporciones de pastos, junto con un aumento de las especies boscosas, los arbustos y las plantas en cojín, que apuntan hacia una mayor heterogeneidad de la vegetación con una composición similar al ecotono bosque-estepa arbustiva que actualmente se desarrolla en este sector del PNPM (Mancini, 2007).

Los trabajos sistemáticos de excavación comenzaron con un sondeo en el área oeste de la cueva (AE1), considerado como el sector de mayor reparo, el cual fue ampliado en diversas campañas arqueológicas. Durante los últimos trabajos de campo se profundizaron las excavaciones en otro sector del sitio ubicado en sector central de la cueva (AE2) (Fig. 2). 


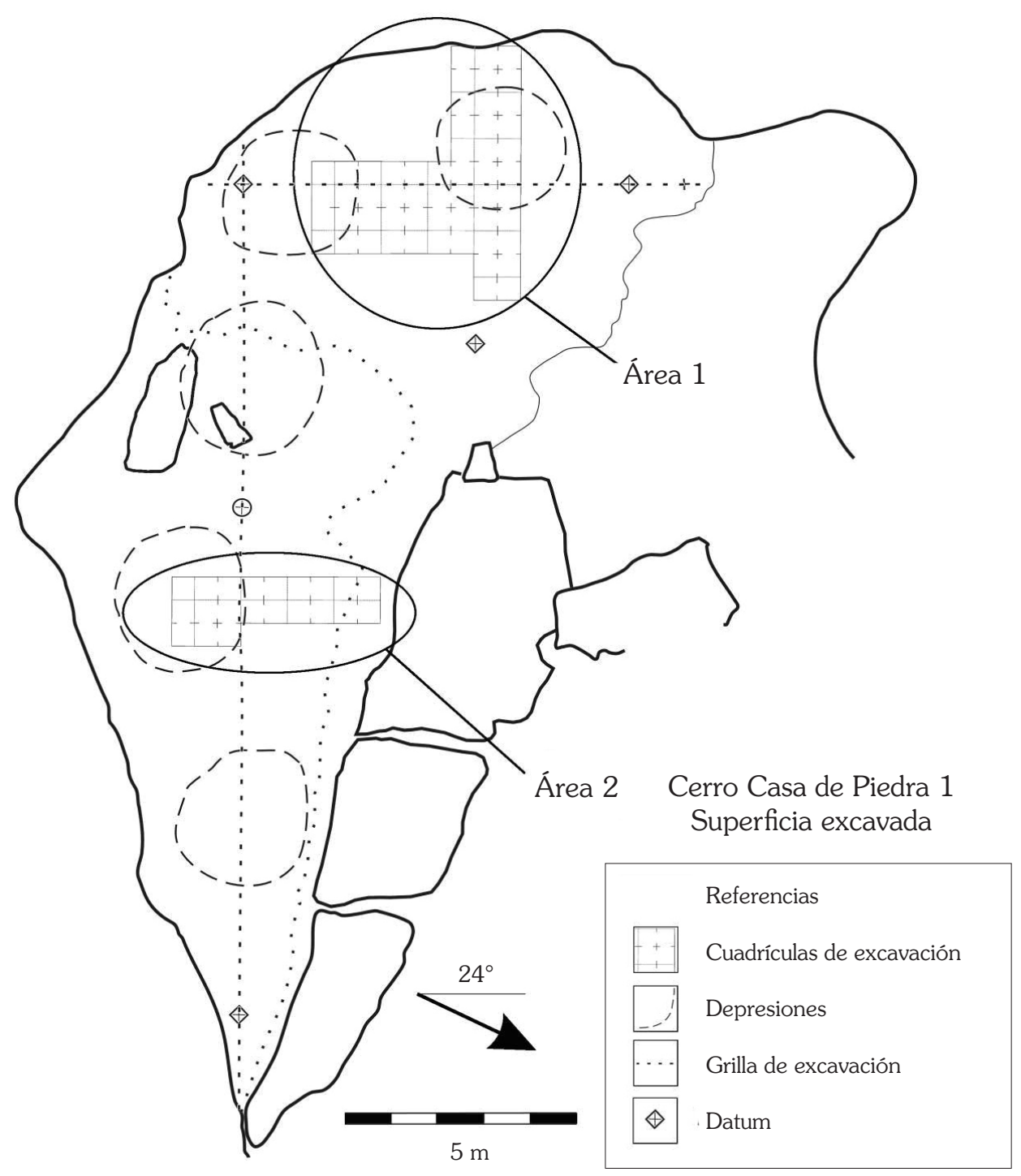

Fig. 2. Planta del sitio Cerro Casa de Piedra 7 y ubicación de las áreas de excavación.

Las tareas desarrolladas en el AE1 permitieron la identificación de una estratigrafía constituida por 18 capas arqueológicas diferenciadas. En este trabajo presentamos exclusivamente los resultados zooarqueológicos de aquella parte de la secuencia que corresponde al Holoceno medio -capas 4, 5 y 6-, datadas aproximadamente en 5.000 años AP. Si bien las características generales de estas capas y las muestras obtenidas han sido presentadas con anterioridad (De Nigris, 2004), en esta ocasión queremos destacar que se observó en este sector una notable redundancia en la ocupación (Aschero, 1996; Aschero et al. 2000) caracterizada, entre otras cosas, por una gran densidad de restos óseos principalmente de guanaco y huemul (Hippocamelus bisulcus); MNI de guanaco elevados; altos porcentajes de marcas de procesamiento; y por la presencia de importantes estructuras de acumulación interpretadas como basurales.

En el AE2 el registro óseo ha sido recuperado en una clara estructura de depositación. Ésta es muy potente y las fechas obtenidas en el inicio y final son estadísticamente similares -ca. 5.000 años AP- (Aschero et al. 2007). Otros atributos también 
Tabla 1. Fórmula dentaria decidua y permanente en guanaco (tomado de Kaufmann, 2009).

\begin{tabular}{|c|c|c|c|c|c|c|c|c|c|c|c|c|c|c|c|}
\hline \multicolumn{7}{|c|}{ Fórmula decidua } & \multicolumn{9}{|c|}{ Fórmula permanente } \\
\hline \multirow{2}{*}{2 ( Id } & 1 & \multirow{2}{*}{$\mathrm{Cd}$} & 1 & \multirow{2}{*}{$\mathrm{Pd}$} & 3 & \multirow{2}{*}{)$=22$} & \multirow{2}{*}{$2(I$} & 1 & \multirow{2}{*}{$\mathrm{C}$} & 1 & \multirow{2}{*}{$\mathrm{P}$} & 2 & \multirow{2}{*}{ M } & 3 & \multirow{2}{*}{)$=30$} \\
\hline & 3 & & 1 & & 2 & & & 3 & & 1 & & 1 & & 3 & \\
\hline
\end{tabular}

indican que se trata de un basural, entre estos podemos mencionar: la gran cantidad de material descartado; el hallazgo de restos con distintos grados de carbonización e incluso de material vegetal sin alteración térmica y la presencia de cenizas. Presenta una frecuencia muy elevada de huesos, principalmente de guanaco y huemul, con altos porcentajes de marcas de procesamiento y consumo (Aschero et al. 2007; Fernández, 2014).

De esta forma, la muestra analizada aquí está compuesta por las mandíbulas y maxilares recuperadas en ambas áreas de excavación -AE1 y AE2-. Si bien el tamaño de las muestras estudiadas aquí varía -e.g. tamaño de la superficie excavadala comparación entre ambos sectores excavados del sitio se encuentra justificada por la similitud estadística de los fechados, sumada a las semejanzas observadas en la densidad de los restos óseos recuperados, así como también en su composición, destacándose los MNI de guanaco elevados y la presencia de todas las partes esqueletarias que conforman el esqueleto.

\section{METODOLOGÍA}

La determinación de la edad de muerte en mandíbulas y maxilares de guanacos fue estimada a partir de la secuencia de erupción y desgaste de las piezas dentales. La dentición de los guanacos se caracteriza por ser heterodonta lo que significa que está compuesta por piezas dentales de morfología y función diferente. De igual modo, se caracteriza por ser difiodonta lo que implica que se encuentra constituida por piezas deciduas y permanentes. En la Tabla 1 se presentan la conformación de la dentición decidua y permanente. Siguiendo a Oporto et al. (1979) y a Kaufmann (2009) 22 piezas constituyen la estructura dental decidua y 30 la permanente. Suelen existir ciertas discrepancias en cuanto a la cantidad de piezas totales, ya que algunos autores consideran la presencia de los $\mathrm{Pd}^{2}$ y $\mathrm{Pd}_{3}$ (Raedeke, 1979), pero se ha demostrado que la incidencia de estas piezas es muy baja, ocurriendo solo entre el $2 \%$ y el $18 \%$ de los casos (Kaufmann, 2009). También se ha planteado una diferencia entre la dentición de machos y hembras, considerando que estas últimas carecen de los caninos superiores. Sin embargo, esta anomalía no ha sido detectada en los casos analizados por Kaufmann (2009).

La estructura dental de este camélido está constituida por seis incisivos y dos caninos deciduos en la parte delantera de la mandíbula. En tanto, la parte anterior del maxilar posee dos incisivos los cuales han adoptado una morfología caniniforme y dos caninos (Oporto et al. 1979; Raedeke, 1979). Estas piezas serán reemplazadas por unas permanentes manteniendo el número de elementos. Al considerar los premolares y molares la situación es más variable. La fórmula dentaria para la mandíbula es la siguiente: el primer y segundo premolar $\left(\mathrm{P}_{1}\right.$ y $\left.\mathrm{P}_{2}\right)$ están ausentes en la mandíbula, el tercer premolar $\left(\mathrm{P}_{3}\right)$ es monocúspide y no será reemplazado posteriormente, el cuarto premolar $\left(\mathrm{Pd}_{4}\right)$ es tricúspide y es sucedido por un premolar $\left(\mathrm{P}_{4}\right)$ monocúspide y tres molares permanentes $\left(M_{1}, M_{2}\right.$ y $\left.M_{3}\right)$, el primer y segundo molar son monocúspides, mientras que el tercero es tricúspide. En tanto que la fórmula para la maxila es la siguiente: solo se encuentra ausente el primer premolar $\left(\mathrm{P}^{1}\right)$, el segundo premolar deciduo $\left(\mathrm{Pd}^{2}\right)$ es monocúspide y no es reemplazado, el tercer y cuarto premolar $\left(\mathrm{Pd}^{3}\right.$ y $\left.\mathrm{Pd}^{4}\right)$ son bicúspides y ambos son sucedidos por premolares monocúspides $\left(\mathrm{P}^{3}\right.$ y $\left.\mathrm{P}^{4}\right)$ y tres molares permanentes bicúspides $\left(\mathrm{M}^{1}\right.$, $\mathrm{M}^{2}$ y $\mathrm{M}^{3}$ ) que no poseen precedentes deciduos (Kaufmann, 2009).

En este trabajo seguimos el cronograma de erupción y desgaste y/o reducción propuesto por Kaufmann (2009). El autor se basó en una muestra conformada por 134 individuos (130 mandíbulas y 118 maxilas) provenientes de una población de guanacos del noreste de la provincia de Río Negro. Si bien la determinación de la edad de muerte no pudo realizarse por medios directos se implementaron una conjunción de métodos 
que permitieron precisar la asignación etaria de los individuos de la muestra. En primer lugar, se ordenó la colección en relación con el desarrollo y desgaste presentes en las mandíbulas. Otro método utilizado fue la aplicación de la técnica de edad establecida en aquellos individuos sobre los que se tenía conocimiento de la fecha de la muerte. Esta técnica es aplicada en especies donde las pariciones se dan en un periodo acotado de tiempo, tal como sucede en el caso del guanaco. Finalmente, se conformaron grupos sobre la base de los lineamientos propuestos por Oporto et al. (1979) y de Lamo (1990).

La asignación a clases y subclases se estableció a partir de la evaluación de los atributos presentes en las piezas dentales tales como la extensión del esmalte, los patrones de exposición de la dentina, morfología de los infundíbulos, categorías de desgaste, estado de las raíces y erupción de piezas permanentes, entre otros. Información más detallada sobre las categorías de desarrollo y desgaste puede ser consultada en Kaufmann (2009).

Ahora bien, es importante mencionar que estamos tratando con variables de intervalo que nos permiten adscribir los elementos óseos a un rango de edad. Una vez determinada la edad de los especímenes se los incluyó dentro del grupo etario correspondiente. La vida promedio del guanaco ronda los 12 años aproximadamente considerándose analíticamente para este lapso las siguientes clases de edad: nonatos (10-11 meses de gestación), crías (0-12 meses), juveniles (1224 meses), subadultos (24-36 meses), adultos (310 años) y seniles (más de 10 años) (Kaufmann, 2009). A su vez estas clases están constituidas por subclases, las cuales varían en función de la resolución brindada por el elemento óseo analizado. Las mandíbulas tienen una resolución más fina ya que la erupción y el desgaste de las piezas dentales es menos variable que en las maxilas. Como resultado de esta diferencia en el desarrollo se cuenta solo con 10 categorías para las maxilas mientras que en las mandíbulas es posible distinguir 19 categorías. Este método de adscripción etaria tiene la virtud de poder aplicarse a lo largo de toda la vida del individuo, aunque como ya hemos precisado, con mayor resolución en las mandíbulas.
Tabla 2. Frecuencia de mandíbulas y maxilares recuperados en CCP7.

\begin{tabular}{cccc}
\hline $\begin{array}{c}\text { Guanaco (Lama } \\
\text { guanicoe) }\end{array}$ & NISP & MANDIBULAS & MAXILARES \\
\hline AE1-Capa 4 & 984 & 8 & 8 \\
\hline AE1-Capa 5 & 278 & 3 & - \\
\hline AE1-Capa 6 & 415 & 3 & 7 \\
\hline AE2 Basural & 927 & 6 & 15 \\
\hline Total & 2.604 & 20 & - \\
\hline
\end{tabular}

\section{RESULTADOS}

De un total de 2.604 especímenes asignados a guanaco hemos identificado 20 mandíbulas y 15 maxilares que conservaban sus piezas dentales de modo tal que posibilitaban la adscripción etaria (Tabla 2).

En relación con la determinación de la edad a partir del análisis de mandíbulas se puede observar que los especímenes se distribuyen en casi todas las clases etarias, a excepción de los seniles (Tabla 3). Dos de ellas fueron registradas como pertenecientes a crías encontrándose representadas las subclases C4 -6 a 9 mesesy C5 -9 a 12 meses-. Para la clase juvenil se contabilizaron cuatro especímenes de los cuales tres exhiben atributos adscribibles a la subclase J1 -12 a 19 meses- y uno a la subclase J2 -19 a 24 meses-. La siguiente clase, subadultos, se encuentra representada por una mandíbula que exhibe características compatibles con la subclase S2 -30 a 36 meses-. Las restantes 12 mandíbulas pertenecen a individuos adultos situados en distintas subclases de esta categoría, destacándose la subclase A4 -6 a 7 años- representada por cinco especímenes.

En cuanto a los maxilares solo se identificaron individuos pertenecientes a las clases subadultos y adultos/seniles (Tabla 4), alcanzado este último grupo un 90,9\%. Dentro de los adultos predominan los maxilares que se ubican en la subclase A2 -4 a 7 años-. Vale decir que estos elementos óseos exhiben una menor variabilidad respecto al conjunto de las mandíbulas.

$\mathrm{Si}$ bien nos basamos en las categorías 
Tabla 3. Estructura de edad en mandíbulas de guanaco de CCP7 (modificado de Kaufmann, 2009).

\begin{tabular}{|c|c|c|c|c|c|c|}
\hline \multicolumn{2}{|c|}{ Clase de edad } & Izquierda & Derecha & MNI & $\%$ & $\%$ \\
\hline \multirow{5}{*}{ Crías } & 0-15 días & - & - & - & - & \multirow{5}{*}{13,3} \\
\hline & 15 días- 3 meses & - & - & - & - & \\
\hline & 3-6 meses & - & - & - & - & \\
\hline & 6-9 meses & 1 & - & 1 & 6,7 & \\
\hline & 9-12 meses & - & 1 & 1 & 6,7 & \\
\hline \multirow{2}{*}{ Juveniles } & $12-19$ meses & 1 & 2 & 2 & 13,3 & \multirow{2}{*}{20} \\
\hline & 19-24 meses & - & 1 & 1 & 6,7 & \\
\hline \multirow{2}{*}{ Subadultos } & 24-30 meses & - & - & - & - & \multirow{2}{*}{6,7} \\
\hline & 30-36 meses & 1 & 1 & 1 & 6,7 & \\
\hline \multirow{7}{*}{ Adultos } & 3-4 años & 2 & 1 & 2 & 13,3 & \multirow{7}{*}{60} \\
\hline & 4-5 años & 2 & 3 & 3 & 19,9 & \\
\hline & 5-6 años & - & - & - & - & \\
\hline & 6-7 años & - & 1 & 1 & 6,7 & \\
\hline & 7-8 años & 1 & - & 1 & 6,7 & \\
\hline & 8-9 años & - & 1 & 1 & 6,7 & \\
\hline & 9-10 años & - & 1 & 1 & 6,7 & \\
\hline \multirow{2}{*}{ Seniles } & 10-11 años & - & - & - & - & \multirow{2}{*}{-} \\
\hline & 11-12 años & - & - & - & - & \\
\hline \multicolumn{2}{|c|}{ MNI } & 8 & 12 & 15 & 100 & 100 \\
\hline
\end{tabular}

Tabla 4. Estructura de edad en maxilares de guanaco de CCP7 (Modificado de Kaufmann, 2009).

\begin{tabular}{|c|c|c|c|c|c|c|}
\hline \multicolumn{2}{|c|}{ Clase de edad } & Izquierda & Derecha & $\mathrm{MNI}$ & $\%$ & $\%$ \\
\hline \multirow{3}{*}{ Crías } & 0-15 días & - & - & - & - & \multirow{3}{*}{ - } \\
\hline & 15 días- 6 meses & - & - & - & - & \\
\hline & 6-12 meses & - & - & - & - & \\
\hline \multirow{2}{*}{ Juveniles } & $12-19$ meses & - & - & - & - & \multirow{2}{*}{-} \\
\hline & $19-30$ meses & - & - & - & - & \\
\hline Subadultos & 30-36 meses & - & 1 & 1 & 9,1 & 9,1 \\
\hline Adultos & 3-4 años & 2 & 1 & 2 & 18,2 & \multirow{3}{*}{90,9} \\
\hline$y$ & 4-7 años & 2 & 6 & 6 & 54,5 & \\
\hline \multirow[t]{2}{*}{ Seniles } & $>7$ años & 2 & 1 & 2 & 18,2 & \\
\hline & & 6 & 9 & 11 & 100 & 100 \\
\hline
\end{tabular}

sugeridas por Kaufmann (2009) debemos señalar que uno de los maxilares recuperados que corresponde a la categoría mayor de 7 años (Tabla 4) exhibe ciertos atributos que nos permiten ubicarlo más cerca del límite inferior de dicha categoría, por lo cual consideramos que no se correspondería con un individuo senil.

\section{DISCUSIÓN Y CONCLUSIONES}

Los perfiles de mortalidad de guanaco obtenidos en CCP7, a partir del análisis de 
las mandíbulas y los maxilares, muestran un predominio de adultos reproductivos. En los guanacos esta etapa de la vida comienza alrededor de los 2 años y finaliza aproximadamente a los 8 años (Kaufmann, 2009). De esto se desprende que las crías, los juveniles y los seniles se encuentran subrepresentados. Esta elección podría responder a las condiciones nutricionales más estables que presentaría esta clase de edad a lo largo del año, a pesar de sufrir oscilaciones estacionales. Por otra parte, consideramos que son los ejemplares adultos los que presentan un mayor rendimiento relativo.

Por otra parte, el perfil de mortalidad reconstruido confirma que el conjunto analizado es el resultado de la caza por los grupos cazadores recolectores dado que la predominancia de animales adultos reproductivos es un claro indicador de esta actividad, distinguiendo claramente a los humanos de otros grandes predadores (Stiner, 1990, 2002; Rivals et al. 2004). Además este tipo de perfil no sería compatible con las estrategias de caza comunal. De ser así, no presentarían esta selección sobre adultos sino que exhibirían un perfil de tipo catastrófico -living structures-, es decir que las edades representadas en la muestra deberían reflejar las proporciones que tienen lugar en una población viva (Stiner, 1990; Rivals et al. 2004; Atici, 2009). Es de destacar que tampoco existen evidencias de este tipo de predación sobre el guanaco en otros contextos de Patagonia (Borrero, 1990, 2013).

Este resultado contradice las expectativas planteadas en función de una ausencia de selectividad del guanaco, como presa principal, para este momento del Holoceno medio -ca. 5.000 años AP- en el que se observaba una mayor redundancia y/o un aumento en la intensidad de la ocupación. Esto parece indicar que este camélido no sufrió una presión de caza significativa durante este periodo que implicara una reducción en su disponibilidad que haya llevado a las poblaciones de cazadores recolectores a ser menos selectivas.

Aunque, como mencionamos anteriormente, existe un predominio de los individuos en la edad reproductiva otros grupos etarios han sido también identificados. Esto señalaría que la predación pudo haberse dirigido hacia los grupos familiares de guanaco, constituidos por un macho adulto, varias hembras y sus crías, generalmente menores a 15 meses (Raedeke, 1979; Franklin, 1982). Además estos grupos sociales se caracterizan por tener hábitos territoriales (Raedeke, 1979; Franklin, 1982), rasgo que los convierte en un recurso más predecible en el paisaje y por tanto pueden convertirse más fácilmente en el foco de la explotación. Aunque los grupos de machos pueden resultar interesantes por el número de individuos que los componen al ser altamente móviles y no tener hábitos territoriales se transforman en un recurso poco predecible en el tiempo y el espacio (Raedeke, 1979; Franklin, 1983; Borrero, 1990, 2013). Al respecto, podemos señalar que en los conjuntos de CCP7 discutidos aquí fue observada con anterioridad la presencia de individuos nonatos (De Nigris, 2004; Fernández, 2014), además de las crías y juveniles identificados aquí, reforzando la idea de que la caza se centró en este grupo social. Una situación similar fue registrada por Rindel (2004) para otros sitios arqueológicos del PNPM.

Es importante aclarar que la ausencia de individuos más jóvenes no respondería a cuestiones relacionadas con la preservación de las muestras. Se ha sugerido que los huesos no fusionados tienen menores probabilidades de supervivencia que aquellos procedentes de individuos adultos (Izeta, 2005; Álvarez et al. 2010), particularidad que podría extenderse a las mandíbulas y maxilares pertenecientes a individuos de distintas edades. Al respecto, debemos destacar la excelente preservación que presentan los materiales óseos procedentes de CCP7 y la baja incidencia de la meteorización (De Nigris, 2004). Por su parte, los valores de correlación entre la densidad ósea y el \%MAU fueron moderados en el AE1 (De Nigris, 2004) y bajos para el AE2 (Fernández, 2014) indicando que la ausencia de estos huesos no podría explicarse por la acción de procesos densitodependientes. La identificación de especímenes atribuibles a individuos nonatos confirma también que las tendencias observadas pueden ser interpretadas como decisiones culturales.

Los datos obtenidos nos posibilitan asimismo hacer apreciaciones sobre la estacionalidad de las ocupaciones de este periodo. Teniendo en cuenta que la parición del guanaco ocurre en una época acotada del año, diciembre a febrero (Larrieu et al. 1979), los elementos analizados sugieren que el 
sitio fue ocupado a lo largo de todo el ciclo anual. Esto podría relacionarse con un tiempo prolongado de ocupación o con ocupaciones repetidas en distintas épocas del año, de modo tal que el sitio formaba parte del circuito de movilidad de las poblaciones en diferentes estaciones. Nuestros resultados son concordantes con los obtenidos por Reigadas (2005, 2007) a partir de los estudios de fibras de huemul y guanaco recuperados en CCP7 para el Holoceno medio. En función de los cambios estacionales que presenta el pelaje de estas especies logró identificar fibras pertenecientes a la muda de otoño y pelaje invernal en el huemul. Asimismo, individualizó vellones de guanaco asignables también a la muda que ocurre a fines de marzo.

Como hemos visto, la construcción de perfiles de mortalidad nos ha permitido avanzar en el conocimiento de las prácticas de caza y la estacionalidad de las ocupaciones de los grupos humanos que habitaron CCP7 durante el Holoceno medio, observando una marcada selectividad de guanacos en edad reproductiva capturados a lo largo de todo el año. De esta forma, no solo se observa una marcada preferencia de los grupos cazadores recolectores por estos ungulados sino también una predilección por aquellos ejemplares que se encuentran en las mejores condiciones nutricionales. El crecimiento del animal está acompañado por cambios en el atractivo nutricional relativo de sus recursos comestibles. En particular las cavidades medulares de los neonatos y de los juveniles muy jóvenes funcionan solamente como sitios de producción de glóbulos rojos, y por tanto resultan pobres en calorías, siendo el cerebro el tejido comparativamente más nutritivo. Los huesos largos de los adultos, sin embargo, se constituyen primariamente como reservorios de grasa para el uso del animal durante períodos de estrés (Blumenschine, 1986; Blumenschine \& Madrigal, 1993). Por su parte, tanto los individuos mayores a dos años como los adultos presentan un rendimiento cárnico relativo que resulta similar (Cunazza, 1978). Además, parece que no existen diferencias significativas entre machos y hembras de camélidos en relación con el porcentaje de carne que brindan (Olivera, 2001). No obstante, una vía que sería interesante explorar en el futuro sería la determinación sexual de las muestras consideradas. Estos resultados son relevantes a la luz de las observaciones realizadas con anterioridad (De Nigris, 2004). El análisis de los restos óseos procedentes del lapso considerado aquí mostró una ausencia de selectividad en el transporte y el aprovechamiento integral de todos los recursos nutritivos aportados por este camélido. Si bien se registra una leve tendencia hacia una mayor representación de las partes anatómicas procedentes del esqueleto apendicular, todas las unidades de la carcasa se encuentran presentes. Elevados porcentajes de marcas de procesamiento y consumo, sumados a las altas frecuencias de fracturas intencionales hablan también de un aprovechamiento integral de esta presa y en especial de un consumo exhaustivo de la médula ósea. Vale decir, entonces, que la preferencia por los individuos adultos podría vincularse también a la necesidad de grasas que tenían estos grupos cazadores-recolectores cordilleranos debido a la mayor proporción de médula presente en los huesos largos y la relativa estabilidad que exhiben a lo largo del ciclo anual.

Resta ahora conocer si los patrones registrados son exclusivos de este periodo o son extensibles a otros momentos temporales. Consideramos entonces que es fundamental la aplicación de esta vía de análisis a otros contextos arqueológicos del sitio y del área para obtener un panorama más amplio de las estrategias de adquisición de esta presa.

\section{AGRADECIMIENTOS}

Este trabajo fue financiado por la ANPCyT en sus proyectos PICT 2006/2488 y 2011/1927 ambos dirigidos por el Lic. Carlos Aschero y por los subsidios UBACyT (20020090200612) Arqueología de la costa noreste del lago Pueyrredón (Santa Cruz), dirigido por María Teresa Civalero y UBACyT (20020100200145), Recursos faunísticosytecnología lítica en la costa noreste del lago Pueyrredón, Santa Cruz, dirigido por Mariana De Nigris.

\section{BIBLIOGRAFÍA}

Álvarez, M., González, M., Massigoge, A., Kaufmann, C., \& Gutiérrez, M. (2010). La densidad mineral ósea y la variabilidad ontogénica en guanaco (Lama guanicoe). Implicancias para la construcción de marcos de 
referencia en Zooarqueología. En M. E. De Nigris, P. M. Fernández, M. Giardina, A. Gil, M. A. Gutiérrez, A. Izeta, G. Neme, \& H. D. Yacobaccio (Eds.), Zooarqueología a principios del siglo XXI: Aportes teóricos, metodológicos y casos de estudio (pp. 95106). Buenos Aires: Libros del Espinillo.

Aschero, C. A. (1996). El Río Belgrano-Lago Posadas (Santa Cruz, Argentina): Problemas y estados de problemas. En Arqueología solo Patagonia. Ponencias de las Segundas Jornadas de Patagonia (pp. 17-26). Puerto Madryn: Centro Nacional Patagónico.

Aschero, C. A., Bellelli, C., \& Goñi, R. (1992). Avances en las investigaciones arqueológicas del Parque Nacional Perito Moreno, Provincia de Santa Cruz, Patagonia Argentina. Cuadernos del Instituto Nacional de Antropología y Pensamiento Latinoamericano, 14, 143-170.

Aschero, C. A., Bellelli, C., Civalero de Biset, M. T., Goñi, R, Guráieb, G., \& Molinari, R. (1992-1993). Cronología y tecnología en el Parque Nacional Perito Moreno (PNPM): ¿Continuidad o reemplazos? Arqueología, 2, 107-134.

Aschero, C. A., Bellelli, C., Civalero de Biset, M. T., Goñi, R., Guráieb, A. G., Molinari, R., \& Espinosa, S. (2005). Holocenic Park: Arqueología del Parque Nacional Perito Moreno. Anales de la Administración de Parques Nacionales. Naturaleza y Cultura, 17, 71-119.

Aschero, C. A., Bozzuto, D, Civalero, M. T., De Nigris, M., Di Vruno, A., Dolce, V., Fernández, N.,...Sacchi, M. (2007). Nuevas evidencias sobre las ocupaciones tempranas en Cerro Casa de Piedra 7. En F. Morello, M. Martinic, A. Prieto, \& G. Bahamonde (Eds.), Arqueología de Fuego-Patagonia. Levantando piedras, desenterrando huesos...y develando arcanos (pp. 569-576). Punta Arenas: Ediciones CEQUA.

Aschero, C. A., Bozzuto, D., Civalero, M. T., De Nigris, M., Di Vruno, A., Dolce, V., Fernández, N.,...Limbrunner, P. (2009). El registro arqueológico de la costa noreste del Lago Pueyrredón-Cochrane (Santa Cruz, Argentina). En M. Salemme, F. Santiago, M. Álvarez, E. Piana, M. Vázquez, \& E. Mansur (Eds.), Arqueología de la Patagonia. Una mirada desde el último confín: Tomo 2 (pp. 919-926). Ushuaia: Editorial Utopías.

Atici, L. (2009). Implications of age structures for Epipaleolithic hunting strategies in the Western Taurus Mountains, Southwest Turkey. Anthropozoologica, 44(1), 13-39. doi.:10.5252/az2009n1a1

Blumenschine, R. J. (1986). Carcass consumption sequences and the archaeological distinction of scavenging and hunting. Journal of Human Evolution, 15, 639-659. doi: 10.1016/S0047-2484(86)80002-1

Blumenschine, R. J., \& Madrigal, T. C. (1993). Variability in long bone marrow yields of east african ungulates and its zooarchaeological implications. Journal of Archaeological Science, 20, 555-587. doi: 10.1006/ jasc. 1993.1034

Borrero, L. A. (1990). Fuego-Patagonian bone assemblages and the problem of comunal guanaco hunting. En L. B. Davis, \& B. O. K. Reeves (Eds.), Hunters of the recent past (pp. 373-399). Londres: Unwin Hyman.

Borrero, L. (2013). Estrategias de caza en Fuego-Patagonia. Comechingonia. Revista de Arqueología, 17, 11-26.

Bourlot, T. (2009). Zooarqueología de sitios a cielo abierto en el lago Cardiel, provincia de Santa Cruz: fragmentación ósea y consumo de grasa animal en grupos cazadores-recolectores del Holoceno tardio. (Tesis inédita de doctorado), Facultad de Filosofía y Letras, Universidad de Buenos Aires, Buenos Aires.

Bourlot, T., Rindel, D., \& Aragone, A. (2009). La fractura transversa/marcado perimetral en sitios a cielo abierto durante el Holoceno tardío en el noroeste de Santa Cruz, En M. Salemme, F. Santiago, M. Álvarez, E. Piana, M. Vázquez, \& E. Mansur (Eds.), Arqueología de la Patagonia. Una mirada desde el último confín Tomo 2 (pp. 693-705). Ushuaia: Editorial Utopías.

Cassiodoro, G., Lublin, G., Piriz, M. F., \& Rindel, D. (2000). Los primeros pasos en el Alero Destacamento Guardaparque: análisis lítico y faunístico (NO Provincia de Santa Cruz, Argentina). En Desde el País de los Gigantes: perspectivas arqueológicas en Patagonia (pp. 369-384). Río Gallegos: Universidad Nacional de la Patagonia Austral.

Cunazza, C. (1978). Rendimiento de carne del guanaco. En K. Raedecke (Ed.), El guanaco de Magallanes, Chile. Su distribución y Biología. Publicación Técnica No 4, Apéndice 2 (pp. 66-174). Chile: Corporación Nacional Forestal. Ministerio de Agricultura.

de Lamo, N. (1990). Determinación de edad en guanacos (Lama Guanicoe Müller) por análisis del desarrollo y desgaste dentario. Veterinaria Argentina, 6 (69), 621-626.

De Nigris, M. E. (2000). Procesando para el consumo: Dos casos de Patagonia meridional. En Desde el País de los Gigantes: Perspectivas arqueológicas en Patagonia (pp. 401-414). Río Gallegos: Universidad Nacional de la Patagonia Austral.

De Nigris, M. E. (2004). El consumo en grupos cazadores recolectores. Un ejemplo zooarqueológico de Patagonia meridional. Buenos Aires: Sociedad 
Argentina de Antropología.

De Nigris, M. E. (2007). Nuevos datos, viejas colecciones: los conjuntos óseos de Cerro Casa de Piedra Cueva 5 (Parque Nacional Perito Moreno, Santa Cruz). Intersecciones en Antropología, 8, 253-264.

De Nigris, M. E. (2008). Modelos de transporte etnoarqueológicos: sobre su aplicabilidad y pertinencia para el interior de Patagonia. En A. Acosta, D. Loponte, \& L. Mucciolo (Comp.) Temas de Arqueología 2. Zooarqueología y Tafonomía (pp. 35-53). Buenos Aires: Instituto Nacional de Antropología y Pensamiento Latinoamericano.

De Nigris, M. E., \& Catá, M. P. (2005). Cambios en los patrones de representación ósea del guanaco en Cerro de los Indios 1 (lago Posadas, Santa Cruz). Intersecciones en Antropología, 6, 109-119.

De Nigris, M. E., \& Tecce, S. (2013). Estudios zooarqueológicos del lago Pueyrredón-Cochrane (Santa Cruz, Argentina)". En A. F. Zangrando, R. Barberena, A. Gil, G. Neme, M. Giardina, L. Luna, C. Otaola, S. Paulides, L. Salgán \& A. Tivoli (Eds.). Tendencias teórico-metodológicas y casos de estudio en la arqueología de la Patagonia (pp. 335-342), Compilado por. Museo de Historia Natural de San Rafael, San Rafael.

Fernández, N. L. (2014). Perfiles de mortalidad en conjuntos arqueofaunísticos holocénicos del Parque Nacional Perito Moreno (Santa Cruz, Argentina): Análisis del sitio Cerro Casa de Piedra 7. (Tesis inédita de Licenciatura). Facultad de Filosofía y Letras, Universidad de Buenos Aires, Buenos Aires.

Franklin, W. L. (1982). Biology, ecology and relationship to man of the South American Camelids, En M. Mares, \& H. Genoway (Eds.), Mammalian Biology in South America (pp. 457-489). Series Pymatuning Laboratory of Ecology, Special Publication 6. Pittsburg: University of Pittsburg.

Gradin, C. J., \& Aguerre, A. M. (Eds.). (1994). Contribución a la arqueología del Río Pinturas, Provincia de Santa Cruz. Concepción del Uruguay: Editorial Búsqueda de Ayllú.

Izeta, A.D. (2005). South American camelid bone structural density: what are we measuring? Comments on data sets, values, their interpretation and application. Journal of Archaeological Science, 32, 1159-1168.

Kaufmann, C. (2004). La fusión ósea como indicador de edad y estacionalidad en guanaco (Lama guanicoe). En M. T. Civalero, P. M. Fernández, \& A. G. Guráieb (Eds.), Contra Viento y Marea. Arqueología de Patagonia (pp. 477-487). Buenos Aires: Instituto Nacional de Antropología y Pensamiento Latinoamericano.
Kaufmann, C. (2009). Estructura de edad y sexo en guanaco. Estudios actualísticos y arqueológicos en Pampa y Patagonia. Buenos Aires: Sociedad Argentina de Antropología.

Larrieu, E., Oporto, N., \& Bigatti, R. (1979). Avances en estudios reproductivos en guanacos del Río Negro (Argentina). Revista argentina de reproducción animal, 3(2), 134-149.

Legge, A., \& Rowly-Conwy, P. (1997). Caza de gacelas en la Siria de la Edad de Piedra. Investigación y Ciencia, 133, 72-80.

Lyman, R. L. (1994). Vertebrate taphonomy. Nueva York: Cambridge University Press.

Mancini, M. V. (2007). Cambios paleoambientales en el ecotono bosque-estepa: análisis polínico del sitio Cerro Casa de Piedra 7, Santa Cruz (Argentina). En F. Morello, M. Martinic, A. Prieto \& G. Bahamonde (Eds.), Arqueología de Fuego-Patagonia. Levantando piedras, desenterrando huesos...y develando arcanos (pp. 89-93). Punta Arenas: Ediciones CEQUA.

Mancini, M. V., Páez, M., \& Prieto, A. (2002). Cambios paleoambientales durante los últimos $7.00014 \mathrm{C}$ años en el ecotono bosque-estepa, 47-48 ${ }^{\circ}$, Santa Cruz, Argentina. Ameghiniana, 39(2), 151-162.

Marchionni, L., Miotti, L., \& Mosquera, B. (2010). El uso de la fauna entre el Pleistoceno final y el Holoceno medio en la Patagonia extra-andina. En M. A. Gutiérrez, M. De Nigris, P. M. Fernández, M. Giardina, A. F. Gil, A. Izeta, G. Neme, \& H. D. Yacobaccio (Eds.), Zooarqueología a principios del siglo XXI: aportes teóricos, metodológicos y casos de estudio (pp. 259271). Buenos Aires: Ediciones del Espinillo.

Mena, F., \& Jackson, D. (1991). Tecnología y subsistencia en Alero Entrada Baker, Región de Aisén, Chile. Anales del Instituto de la Patagonia (Serie Ciencias Sociales), 20, 169-203.

Méndez, C., Reyes, O., Nuevo Delaunay, A., Trejo, V., Barberena, R., \& Velásquez, H. (2011). Ocupaciones humanas en la margen occidental de Patagonia Central: eventos de poblamiento en alto río Cisnes. Magallania, 39 (2), 223-242.

Mengoni Goñalons, G. L. (1988). Análisis de materiales faunísticos de sitios arqueológicos. Xama, 1, 71-120.

Mengoni Goñalons, G. L. (1999). Cazadores de guanacos de la estepa patagónica. Buenos Aires: Sociedad Argentina de Antropología.

Miotti, L. (1998). Zooarqueología de la meseta central y costa de Santa Cruz. Un enfoque de las estrategias adaptativas aborígenes y los paleoambientes. San 
Rafael, Museo de Historia Natural.

Miotti, L. (2006). Paisajes domésticos y sagrados desde la arqueología de los cazadores- recolectores en el macizo del Deseado, provincia de Santa Cruz. Cazadoresrecolectores del Cono Sur, 1, 11-40.

Miotti, L., Vázquez, M., \& Hermo, D. (1999). Piedra Museo, un Yamnagoo pleistocénico de los colonizadores de la meseta de Santa Cruz. El estudio de la arqueofauna. En Soplando en el viento: Actas de las Terceras Jornadas de la Arqueología de la Patagonia (pp. 113136). Neuquén/Buenos Aires: Universidad Nacional del Comahue e Instituto Nacional de Antropología y Pensamiento Latinoamericano.

Olivera, D. E. (2001). Perfil etario y rendimiento económico de Lama glama. En G. L. Mengoni Goñalons, D. E. Olivera, \& H. D. Yacobaccio (Eds.), Zooarqueología de camélidos 3: el uso de los camélidos a través del tiempo (pp. 179-202). Buenos Aires: Grupo Zooarqueología de Camélidos.

Oporto, N., Bigatti, R., \& Larrieu, E. (1979). Determinación de edades en guanaco (Lama guanicoe) en base a su dentición. Revista Argentina de producción animal, 4(9), 965-983.

Raedeke, K. J. (1979). Population dynamics and sociology of the guanaco (Lama guanicoe) of Magallanes, Chile. (Tesis inédita de doctorado). Seattle: Washington University.

Redford, K. H., \& Eisenberg, J. F. (1992). Mammals of the Neotropics. The Southern Cone, Volume 2: Chile, Argentina, Uruguay, Paraguay. Chicago/Londres: The University of Chicago Press.

Reigadas, M. C. (2005). Fibras arqueológicas de origen animal. Análisis microscópico de muestras de fibras de Cerro Casa de Piedra -CCP5 y CCP7- (Santa Cruz, Argentina). Relaciones de la Sociedad Argentina de Antropología, 30, 235-243.

Reigadas, M. C. (2007). Cazadores del Holoceno y los recursos faunísticos. Estudio de fibras animales de Cerro Casa de Piedra-CCP5 y CCP7 (Santa Cruz, Argentina). En F. Morello, M. Martinic, A. Prieto, \& G. Bahamonde (Eds.), Arqueología de Fuego-Patagonia. Levantando piedras, desenterrando huesos...y develando arcanos (pp. 663-674). Punta Arenas: Ediciones CEQUA.

Rindel, D. (2003). Patrones de procesamiento faunístico durante el Holoceno medio y tardio en el sitio Alero Destacamento Guardaparque (Parque Nacional Perito Moreno, provincia de Santa Cruz, Argentina). (Tesis inédita de Licenciatura). Facultad de Filosofía y Letras, Universidad de Buenos Aires, Buenos Aires.
Rindel, D. (2004). Patrones de procesamiento faunístico en el sitio Alero Destacamento Guardaparque durante del Holoceno medio. En M. T. Civalero, P. M. Fernández, \& A. G. Guráieb (Eds.), Contra Viento y Marea. Arqueología de Patagonia (pp. 263-276). Buenos Aires: Instituto Nacional de Antropología y Pensamiento Latinoamericano.

Rindel, D. (2008). Arqueología de momentos tardíos en el noroeste de la provincia de Santa Cruz (Argentina): una perspectiva faunística. (Tesis inédita de Doctoral). Facultad de Filosofía y Letras, Universidad de Buenos Aires, Buenos Aires.

Rindel, D. (2013). Arqueofaunas del Holoceno medio y tardío de sectores altos del noroeste de la provincia de Santa Cruz. En A. F. Zangrando, R. Barberena, A. Gil, G. Neme, M. Giardina, L. Luna, C. Otaola, S. Paulides, L. Salgán, \& A. Tivoli (Eds.), Tendencias teóricometodológicas y casos de estudio en la arqueología de la Patagonia (pp. 505-513). San Rafael: Museo de Historia Natural de San Rafael.

Rivals, F., Kacimi, S., \& Moutoussamy, J. (2004). Artiodactyls, favorite game of prehistoric hunters at the Caune de L'Arago Cave (Tautavel, France). Opportunistic or selective hunting strategies? European Journal of Wildlife Research, 50, 25-32.

Savanti, F., Bourlot, T., \& Aragone, A. (2004). Arqueofauna y ocupación tardía de las franjas de médanos del lago Cardiel, provincia de Santa Cruz. En M. T. Civalero, P. M. Fernández, \& A. G. Guráieb (Eds.), Contra viento y marea. Arqueología de Patagonia (pp. 497-506). Buenos Aires: Instituto Nacional de Antropología y Pensamiento Latinoamericano.

Silveira, M. J. (1979). Análisis e interpretación de los restos faunísticos de la Cueva Grande de Arroyo Feo (Provincia de Santa Cruz). Relaciones de la Sociedad Argentina de Antropología, 13, 229-247.

Stiner, M. C. (1990). The Use of Mortality Patterns in Archaeological Studies of Hominid Predatory Adaptations. Journal of Anthropological Archaeology, 9, 305-351.

Stiner, M. C. (2002). Carnivory, coevolution, and the geographic spread of the genus Homo. Journal of Archaeological Research, 10 (1), 2-63.

Yacobaccio, H., \& Guráieb, A. G. (1994). Tendencia temporal de contextos arqueológicos: área del Río Pinturas y zonas vecinas. En C. J. Gradin, \& A. M. Aguerre (Eds.), Contribución a la arqueología del río Pinturas, provincia de Santa Cruz (pp. 13-28). Concepción del Uruguay: Búsqueda de Ayllú. 